\title{
Freqüência de enteroparasitas em amostras fecais de cães e gatos dos municípios do Rio de Janeiro e Niterói
}

\section{Frequency of enteric parasites in fecal samples of dogs and cats in Rio de Janeiro and Niterói}

\author{
Brener,B., ${ }^{*}$ Lisboa, L., ${ }^{* *}$ Mattos, D.P.B.G., ${ }^{* *}$ Arashiro, E.K.N., ${ }^{* * *}$ Millar, P.R., ${ }^{* * *}$ Sudré, A.P., ${ }^{* * *}$ Duque, V. ${ }^{* * *}$
}

\begin{abstract}
Resumo
Foram analisadas 252 amostras de fezes de cães e gatos provenientes dos municípios do Rio de Janeiro e Niterói no período de 1999 a 2000. Foram $212(84,12 \%)$ amostras de cães e $40(15,87 \%)$ de gatos. Do total de amostras examinadas 81 (32,14\%), foram positivas para pelo menos uma espécie de parasita. Dos 212 exames de material fecal canino, $70(33,01 \%)$ estavam positivos e de felinos $11(27,5 \%)$. As infecções mistas foram observadas em 10 amostras caninas $(14,28 \%)$ e quatro felinas $(36,36 \%)$. Nas amostras caninas, ovos de ancilostomídeos foram os mais encontrados em $43(61,43 \%)$ amostras, enquanto os coccídeos foram mais freqüentemente observados nas fezes de felinos em três $(27,27 \%)$ amostras. Quanto à idade dos animais, 37 (52,85\%) amostras positivas eram provenientes de animais com mais de 1 ano de idade, indicando provável vermifugação dos filhotes, enquanto nos gatos a faixa etária mais parasitada foi de menos de seis meses, com 7 $(63,63 \%)$ positivos.
\end{abstract}

Palavras-chave: enteroparasitas, cães, gatos, fezes.

\begin{abstract}
252 fecal samples from dogs and cats were analysed. 212 of the samples (84.12\%), were from dogs and 40 samples (15.87\%) were from cats. Of all of the samples examined, $81(32.14 \%)$ were positive for at least one parasite. 70 of the 212 exams (33.01\%) of fecal samples from dogs were positive, while 11 of the 40 samples $(27.5 \%)$ from cats were positive. In $10(14.28 \%)$ dog samples and $4(36.36 \%)$ cat samples, multiple infections were found. Hookworm eggs were found in $43(61,43 \%)$ dog samples, being the most common among all parasites. The coccidians were more frequently found in the cat samples, in a total number of $3(27,27 \%)$. Regarding the age of the animals, $37(52,85 \%)$ positive dog samples were collected from animals at least one year old, indicating a possible treatment of the puppies. On the other hand, among the cats, the more affected were those aged less than 6 months, with 7 (63,63\%) positive samples.
\end{abstract}

Keywords: intestinal parasites, dogs, cats, feces.

\section{Introdução}

As parasitoses intestinais são bastante freqüentes entre os animais, tanto em áreas rurais quanto urbanas, por todo o mundo (Cortes et al., 1988; Malgor et al., 1996; El-Shehabi et al., 1999; Choo et al., 2000). Estas podem causar diferentes sintomas clínicos, dependendo da espécie e quantidade de parasitos. Os sintomas mais comuns são gastrintestinais, como anorexia e perda de peso, anemia e desidratação. Em casos mais severos, deficiências do crescimento e até a morte podem ocorrer (Barutzki e Schaper, 2003). Alguns parasitas que acometem animais domésticos são também agentes causadores de enfermidades em humanos, mostrando, assim, um caráter zoonótico. Os principais enteroparasitas de cães e gatos são responsáveis pelas enfermidades huma- nas Larva Migrans Visceral (LMV) por ovos de Toxocara sp, Larva Migrans Cutânea (LMC) por larvas de ancilostomídeos e enterite eosinofílica (EE) por larvas de Ancylostoma caninum (Tan, 1997; Overgaauw, 1997).

O homem adquire a LMV através da ingestão de ovos contendo a larva de segundo estágio do Toxocara sp. Esta, após eclodir do ovo, penetra na parede do intestino e inicia uma migração para o fígado, pulmão e outros órgãos. Os sinais e sintomas podem variar bastante, dependendo da localização da larva, sendo os mais comuns febre, problemas respiratórios e hepatomegalia (Martinez-Barbabosa et al., 2003; Anaruma Filho et al., 2003). Em um estudo realizado em Campinas, SP, por Anaruma Filho et al. (2003), foi encontrado $20,9 \%$ de positividade para anticorpos anti-Toxocara no sangue dos indivíduos analisados.

\footnotetext{
* Professor adjunto da disciplina de Parasitologia da Universidade Federal Fluminense

** Professor substituto da disciplina de Parasitologia da Universidade Federal Fluminense

*** Monitores da disciplina de Parasitologia da Universidade Federal Fluminense
} 
A LMC manifesta-se quando larvas de ancilostomídeos penetram na pele do homem e vagueiam no tecido subcutâneo provocando uma erupção linear e tortuosa da pele, geralmente muito pruriginosa (Nunes et al., 2000).

A EE ocorre quando há ingestão de larvas de terceiro estágio (L3) de Ancylostoma caninum, que é um parasita comum em cães de regiões tropicais, onde esta patologia se mostra também bem elevada (Robertson et al., 2000).

As crianças são consideradas grupo de risco para o desenvolvimento de tais enfermidades, principalmente pelo contato mais freqüente com o solo de parques e áreas públicas, como também pelo hábito de geofagia (Martinez-Barbabosa et al., 2003).

O crescente número de cães domiciliados, peridomiciliados e errantes, de modo geral, em todo o Brasil, associado ao fácil acesso destes animais a locais de lazer, aumenta o risco de infecção, especialmente para as crianças (Scaini et al., 2003). Sendo assim, o conhecimento da prevalência e incidência dos parasitas de animais domésticos que possuem caráter zoonótico é fundamental para o planejamento e instituição de medidas profiláticas, visando tanto o bem-estar e a sanidade animal quanto a saúde publica.

Com a finalidade de trazer algum conhecimento sobre as enteroparasitoses de cães e gatos dos municípios do Rio de Janeiro e de Niterói, foi realizado um levantamento sobre a freqüência e associações parasitárias encontradas. $O$ presente estudo foi baseado em resultados de exames realizados rotineiramente no Laboratório de Parasitologia do Departamento de Microbiologia e Parasitologia da Universidade Federal Fluminense (UFF) no período de 1999 a 2002.

\section{Material e métodos}

Durante o período de 1999 a 2002, foram analisadas 252 amostras fecais de cães e gatos pertencentes a membros da comunidade dos municípios do Rio de Janeiro e Niterói. Estas amostras foram encaminhadas rotineiramente ao Laboratório da disciplina de Parasitologia da Universidade Federal Fluminense. Cada amostra fecal foi processada utilizando-se duas técnicas com fundamentos distintos, sendo uma de sedimentação espontânea (Hoffman et al., 1934) e outra de flutuação em solução de sulfato de zinco com densidade igual a 1,180 (Faust et al., 1938). As amostras foram remetidas sob conservação química (em solução de Ralliet e Henry) ou física (sob refrigeração), sendo as últimas processadas em até 24 horas após a coleta.

Os animais envolvidos no estudo constituíram uma amostra bastante heterogênea com representantes de diversas idades, raças e sexos.

\section{Resultados}

Dos 252 exames coproparasitológicos realizados, 212 $(84,12 \%)$ eram referentes a amostras de cães e $40(15,87 \%)$ de gatos. Do total examinado, $81(32,14 \%)$ foram positivos para pelo menos uma espécie de parasita. Dos 212 exames de material fecal canino, $70(33,01 \%)$ foram positivos e 11 $(27,5 \%)$ no caso dos felinos. Infecções mistas foram observadas em 10 amostras caninas $(14,28 \%)$ e quatro felinas $(36,36 \%)$. Nos exames de fezes de cães, os ovos de ancilostomídeos foram as formas parasitárias mais freqüentes, estando presentes em 43 amostras $(61,43 \%)$. No caso dos felinos, os coccídeos foram as formas mais encontradas, correspondendo a três resultados positivos (27,27\%).

Com relação à idade dos animais, 37 (52,85\%) das amostras positivas de cães eram provenientes de animais com mais de 1 ano, sendo que sete não tiveram suas idades definidas. No que se refere aos felinos, sete $(63,63 \%)$ amostras positivas eram de animais com menos de 1 ano de idade, e uma amostra não havia idade relacionada.

Os resultados estão resumidos nas Tabelas 1 a 6 .

Tabela 1 - Distribuição dos resultados quanto à espécie animal analisada em amostras fecais de cães e gatos dos municípios do Rio de Janeiro e Niterói nos anos de 1999 e 2000

\begin{tabular}{ccccccc} 
& \multicolumn{2}{c}{ CÃES } & \multicolumn{2}{c}{ GATOS } & \multicolumn{2}{c}{ TOTAL } \\
\hline AMOSTRAS & No. & $\%$ & No. & $\%$ & No. & $\%$ \\
\hline Positivas & 70 & 33,0 & 11 & 27,5 & 81 & 32,1 \\
Negativas & 142 & 67,0 & 29 & 72,5 & 171 & 67,9 \\
\hline TOTAL & 212 & 100,0 & 40 & 100,0 & 252 & 100,0 \\
\hline
\end{tabular}

Tabela 2 - Resultados dos exames coproparasitológicos em amostras de cães dos municípios do Rio de Janeiro e Niterói nos anos de 1999 e 2000 de acordo com a faixa etária dos animais

Até 6 meses $\quad$ De 6 meses a 1 ano $\quad$ Acima de 1 ano $\quad$ Sem $\quad$ TOTAL

idade

definida

\begin{tabular}{lccccc}
\hline Positivo & 17 & 9 & 37 & 7 & 70 \\
& $(24,29 \%)$ & $(12,86 \%)$ & $(52,85 \%)$ & $(10,00 \%)$ & $(100,0 \%)$ \\
\multirow{2}{*}{ Negativo } & 27 & 16 & 97 & 2 & 142 \\
& $(19,01 \%)$ & $(11,27 \%)$ & $(68,31 \%)$ & $(1,41 \%)$ & $(100,0 \%)$ \\
\hline
\end{tabular}


Tabela 3 - Resultados dos exames coproparasitológicos em amostras de gatos dos municípios do Rio de Janeiro e Niterói nos anos de 1999 e 2000 de acordo com a faixa etária dos animais

\begin{tabular}{cccccc} 
& Até 6 meses & De 6 meses a 1 ano & Acima de 1 ano & $\begin{array}{c}\text { Sem } \\
\text { idade } \\
\text { definida }\end{array}$ & TOTAL \\
\hline Positivo & 7 & 0 & \multicolumn{3}{c}{11} \\
Negativo & $(63,63 \%)$ & $(0,0 \%)$ & $(27,28 \%)$ & $(9,09 \%)$ & $(100,0 \%)$ \\
& $(31,03 \%)$ & $(6,90 \%)$ & $(51,72 \%)$ & $(10,35 \%)$ & $(100,0 \%)$ \\
\hline
\end{tabular}

\section{Discussão}

Analisando-se a idade dos animais, 37 (52,85\%) amostras positivas de cães eram referentes a animais maiores de 1 ano, indicando provável vermifugação dos filhotes, enquanto nos gatos a faixa etária mais freqüentemente parasitada foi de menos de 6 meses, com $7(63,63 \%)$ positivos. A maioria das amostras examinadas foi proveniente de ani-

Tabela 4 - Distribuição de parasitismo simples ou múltiplo na população amostral dos municípios do Rio de Janeiro e Niterói nos anos de 1999 e 2000

\begin{tabular}{ccccc} 
& \multicolumn{2}{c}{ CÃES } & \multicolumn{2}{c}{ GATOS } \\
\hline INFECÇÃO & № & $\%$ & № & $\%$ \\
\hline Simples & 60 & 85,7 & 7 & 63,6 \\
Dupla & 7 & 10,0 & 3 & 27,3 \\
Tripla & 3 & 4,3 & 1 & 9,1 \\
\hline TOTAL & 70 & 100,0 & 11 & 100,0 \\
\hline
\end{tabular}

mais que pertenciam a pessoas que conheciam os perigos das parasitoses e dispunham de recursos para encaminhar seus animais a um médicoveterinário assim como comprar medicamentos. Apesar disso, 32,14\% de amostras de cães e gatos foram positivas sendo este um número bastante significativo do ponto de vista médico e sanitário, sugerindo que em comunidades carentes estes índices sejam ainda superiores e colocando em risco a saúde animal e humana, já que alguns parasitas têm caráter zoonótico.

Os resultados mostraram um número expressivo de animais parasitados. Diversos autores, analisando animais domiciliados ou não, também obtiveram percentual elevado de positividade em diversas regiões do Brasil e em outros países (Fenerich et al., 1972; Carneiro et al.,1973; Hatschbach et al., 1976; Lara et al.,1981; Carlo et al.,1983; Araújo et al., 1986; Côrtes et al., 1988; Malgor et al., 1996; e Taranto et al., 2000; Labarthe et al., 2004). Os ancilostomídeos foram os principais causadores de infecção nos cães de todas as faixas etárias $(20,3 \%)$, fato observado também por Fenerich et al. (1972); Hatschbach et al. (1976); Carlo et al. (1983); Araújo et al. (1986); Taranto et al. (2000); e Eguía-Aguilar et al. (2005), e diferentemente de Overgaauw (1997), que não encontrou ovos de ancilostomídeos.

A segunda espécie mais encontrada no presente estudo foi o Toxocara canis, observado em $7,1 \%$ das amostras provenientes de cães, estando de acordo com as observações de Araújo et al. (1986) e Oliveira et al. (1990). A prevalência em gatos sofreu significativa redução quando os animais apresentaram mais de seis meses de idade.

Os coccídeos foram observados em $7,1 \%$ dos cães, resultados superiores ao de Fenerich et al. (1972) que foi de apenas $1,3 \%$.

Tabela 6 - Principais associações parasitárias encontradas em amostras fecais de cães e gatos dos municípios do Rio de Janeiro e Niterói nos anos de 1999 e 2000

\begin{tabular}{lcccccc}
\cline { 2 - 7 } & \multicolumn{2}{c}{ Cães } & \multicolumn{2}{c}{ Gatos } & \multicolumn{2}{c}{ Total } \\
\hline \multicolumn{1}{c}{ ASSOCIAÇÃO PARASITÁRIA } & No. & $\%$ & No. & $\%$ & No. & $\%$ \\
\hline Ancilostomídeo, Toxocara sp & 3 & 30 & 3 & 75 & 6 & 42,9 \\
Ancilostomídeo, Coccídeo & 2 & 20 & 0 & 0 & 2 & 14,4 \\
Ancilostomídeo. Dipylidium caninum & 1 & 10 & 0 & 0 & 1 & 7,1 \\
Ancilostomídeo, Toxocara sp, Coccídeo & 2 & 20 & 1 & 25 & 3 & 21,4 \\
Ancilostomídeo, Giardia sp & 1 & 10 & 0 & 0 & 1 & 7,1 \\
Ancilostomídeo, Coccídeo, Trichuris vulpis & 1 & 10 & 0 & 0 & 1 & 7,1 \\
\hline TOTAL & 10 & 100,0 & 4 & 100,0 & 14 & 100,0
\end{tabular}

A principal associação parasitária encontrada foi de ancilostomídeos e Toxocara canis, também observada por Araújo et al. (1986) e Oliveira et al. (1990), diferentemente de Fenerich et al. (1972) e Lara et al. (1981), que diagnosticaram a principal associação entre ancilostomídeos e Trichuris vulpis. A alta freqüência de ancilostomídeos e Toxocara canis observada em animais de companhia é preocupante, uma vez que são agentes da LMC e LMV, no homem.

Diante dos resultados obtidos no presente trabalho conclui-se uma significativa presença de animais 
parasitados. Tal fato é preocupante considerando-se que estes animais eram domiciliados e viviam em condições higiênico-sanitárias adequadas. Este resultado pode estar diretamente associado a falhas no tratamento profilático des-

\section{Referências}

ANARUMA FILHO, F.; CHIEFFI, P. P.; CORREA, C. R. S.; CAMARGO, E. D.; SILVEIRA, E. P. R.; ARANHA, J. J. B. Human toxocariasis: incidence among residents in the outskirts of Campinas, state of São Paulo, Brazil. Rev. Inst. Med. Trop. São Paulo, v. 45, n. 5, p. 293-294, 2003. ARAUJO, R. B.; FERREIRA, P. M.; ASSIS, C. B.; DEL CARLO, R.J . Helmintoses intestinais em cães da microrregião de Viçosa - Minas Gerais. Arq. Bras. Med. Vet. Zoot., v. 38, n. 2, p. 197-203, 1986.

BARUTZKI, D.; SCHAPER, R. Endoparasites in dogs and cats in Germany 1999-2002. Parasitol. Res., v. 90, p. S148-S150, 2003.

CARLO, R. J. D.; ARAÚJO, R. B.; ASSIS, C. B. Avaliação preliminar das helmintíases em cães do Município de Viçosa, Minas Gerais. Rev. Ceres., v. 30, n. 171, p. 388-390, 1983.

CARNEIRO, J. R., FREITAS, J. S., PEREIRA, E.; CAMPOS, D. M. B.; JARDIM, C. V. Prevalência de helmintos em "Canis familiaris" no Município de Goiânia. Rev. Patol. Trop., v. 4, n.2, p. 401-404, 1973.

CHOO, J.; PANG, E.; PROCIV, P. Hookworms in dogs of Kuching, Sarawak (north Borneo). Trans. R. Soc. Trop. Med. Hyg., v. 94, n.1, p. 21-22, 2000.

CÔRTES, V. A.; PAIM, G. V.; ALENCAR FILHO, R. A. Infestações por ancilostomídeos e toxocarídeos em cães e gatos apreendidos em vias públicas, São Paulo (Brasil). Rev. Saúde Públ., v. 22, n. 4, p. 341-343, 1988.

EGUÍA-AGUILAR, P.; CRUZ-REYES, A.; MARTÍNEZ-MAYA, J. J. Ecological analysis and description of the intestinal helminths present in dogs in Mexico City. Veterinary Parasitology, v. 127, p. 139-146, 2005.

EL-SHEHABI, F. S.; ABDEL-HAFEZ, S. K., KAMHAWI, S. A. Prevalence of intestinal helminthes of dogs and foxes from Jordan. Parasitol. Res., v. 85, n. 11, p. 928-934, 1999.

FAUST, E. C.; D'ANTONI, J. S.; ODOM, V.; MILLER, M. J.; PERES, C.; SAWITZ, W.; THOMEN, L. F.; TOBIE, J.; WALKER, J. H. A critical study of clinical laboratory thecnics for the diagnosis of protozoan cysts and helminth eggs in feces. I. Prelimynary communication. Amer. J. Trop. Med., v. 18, p. 169-183, 1938.

FENERICH, F. L., SANTOS, S. M.; AMARAL, V. Análises dos resultados obtidos em 903 amostras de fezes oriundas da espécie canina. $O$ Biológico, v. 38, p. 175-177, 1972.

HATSCHBACH, P. I.; RIBEIRO, R.; RIBEIRO, L. A. R. Ocorrência de endoparasitas em cães da cidade do Rio de Janeiro. Ci. e Cult., v. 28, n. 7, p. 509, 1976. tes enteroparasitas, assim como à reinfecção pelo fato destes freqüentarem locais onde não há restrição ao acesso dos animais errantes possibilitando a perpetuação da contaminação ambiental.

HOFFMAN, W. A.; PONS, J.A., JANER, J.L. The sedimentation method in schistosomiasis mansoni. The Puerto Rico J. Publi. Health Trop. Med., v. 9, n. 3, p.283-291, 1934.

LABARTHE, N., SERRÃO, M. L.; FERREIRA, A. M. R.; ALMEIDA, N. K. O.; GUERRERO, J. A survey of gastrointestinal helminths in cats of the metropolitan region of Rio de Janeiro, Brazil. Veterinary Parasitology, v. 123, p. 133-139, 2004.

LARA, S. I. M.; TAROUCO, M. R. R.; RIBEIRO, P. B. Helmintos parasitos de Canis familiaris de Pelotas - Rio Grande do Sul. Arq. Bras. Med. Vet. Zoot., v. 33, n. 2, p. 293-297, 1981.

MALGOR, R.; OKU, Y.; GALLARDO, R.; YARZÁBAL, L. High prevalence of Ancylostoma spp. infection in dogs, associated with endemic focus of human cutaneous Larva Migrans, in Tacuarembo, Uruguay. Parasite, v. 3, p. 131-134, 1996.

MARTINEZ-BARBABOSA, I.; TSUJI, O. V.; CABELLO, R. R.; CÁRDENAS, E. M. G.; CHASIN, O. A. The prevalence of Toxocara cati in domestic cats in Mexico City. Veterinary Parasitology, v. 114, p. 43-49, 2003.

NUNES, C. M.; PENA, F. C.; NEGRELLI, G. B.; ANJO, C. G. S.; NAKANO, M. M.; STOBBE, N. S. Ocorrência de larva migrans na areia de áreas de lazer das escolas municipais de ensino infantil, Araçatuba, SP, Brasil. Rev. Saúde Pública, v. 34, n. 6, p. 656-658, 2000.

OLIVEIRA, P. R.; SILVA, P. L.; PARREIRA, V. F.; RIBEIRO, S. C.A.; GOMES, J. B. Prevalência de endoparasitos em cães da Região de Uberlândia, Minas Gerais. Braz. J. Vet. Res. Anim. Sci., v. 27, n. 2, p. 193-197, 1990.

OVERGAAUW, P.A. Prevalence of intestinal nematodes of dogs and cats in the Netherlands. The Veterinary quarterly. v. 19, n.1, p. 14$17,1997$.

ROBERTSON, I. D.; IRWIN, P. J.; LYMBERY, A. J.; THOMPSON, R. C. A. The role of companion animals in the emergence of parasitic zoonoses. Int. J. Parasitol., v. 30, p. 1369-1377, 2000.

SCAINI, C. J.;TOLEDO, R. N.; LOVATEL, R.; DIONELLO, M. A.; GATTI, F. A.; SUSIN, L.; SIGNORINI, V. R. M. Contaminação ambiental por ovos e larvas de helmintos em fezes de cães na área central do Balneário Cassino, Rio Grande do Sul. Rev. Soc. Bras. Med. Trop., v. 36, n. 5, p. 617-619, 2003.

TAN, J. S. Human zoonotic infections transmitted by dogs and cats. Arch. Intern. Med., v. 157, n. 17, p. 1933-943, 1997.

TARANTO, N. J.; PASSAMONTE, L.; MARINCONZ, R.; MARZI, M. C.; CAJAL, S. P.; MALCHIODI, E. L. Parasitosis zoonoticas transmitidas por perros en el Chaco Salteño. Medicina (Buenos Aires), v. 60, p. 217-220, 2000. 